\title{
Circular RNA Signature Predicts Gemcitabine Resistance of Pancreatic Ductal Adenocarcinoma
}

\section{OPEN ACCESS}

Edited by:

Marilena Valeria Iorio, Istituto Nazionale dei Tumori (IRCCS),

Reviewed by:

Paul B. Fisher,

Virginia Commonwealth University,

United States

Chun Hei Antonio Cheung, National Cheng Kung University,

Taiwan

${ }^{*}$ Correspondence:

Qiang Huang ahslyyhq2016@163.com

tThese authors have contributed equally to this work.

Specialty section:

This article was submitted to Cancer Molecular Targets and Therapeutics, a section of the journal

Frontiers in Pharmacology

Received: 12 January 2018

Accepted: 16 May 2018

Published: 05 June 2018

Citation:

Shao F, Huang M, Meng F and

Huang Q (2018) Circular RNA Signature Predicts Gemcitabine Resistance of Pancreatic Ductal Adenocarcinoma.

Front. Pharmacol. 9:584. doi: 10.3389/fphar.2018.00584
Feng Shao ${ }^{1,2+}$, Mei Huang ${ }^{2 \dagger}$, Futao Meng ${ }^{2}$ and Qiang Huang ${ }^{1,2 *}$

${ }^{1}$ Medical College, Shandong University, Jinan, China, ${ }^{2}$ Anhui Province Key Laboratory of Hepatopancreatobiliary Surgery, Department of General Surgery, Anhui Provincial Hospital, Hefei, China

Gemcitabine resistance is currently the main problem of chemotherapy for advanced pancreatic cancer patients. The resistance is thought to be caused by altered drug metabolism or reduced apoptosis of cancer cells. However, the underlying mechanism of Gemcitabine resistance in pancreatic cancer remains unclear. In this study, we established Gemcitabine resistant PANC-1 (PANC-1-GR) cell lines and compared the circular RNAs (circRNAs) profiles between PANC-1 cells and PANC-1-GR cells by RNA sequencing. Differentially expressed circRNAs were demonstrated using scatter plot and cluster heatmap analysis. Gene ontology and pathway analysis were performed to systemically map the genes which are functionally associated to those differentially expressed circRNAs identified from our data. The expression of the differentially expressed circRNAs picked up by RNAseq in PANC-1-GR cells was further validated by GRT-PCR and two circRNAs were eventually identified as the most distinct targets. Consistently, by analyzing plasma samples form pancreatic ductal adenocarcinoma (PDAC) patients, the two circRNAs showed more significant expression in the Gemcitabine non-responsive patients than the responsive ones. In addition, we found that silencing of the two circRNAs could restore the sensitivity of PANC-1-GR cells to Gemcitabine treatment, while over-expression of them could increase the resistance of normal PANC-1 and MIA PACA-2 cells, suggesting that they might serve as drug targets for Gemcitabine resistance. Furthermore, the miRNA interaction networks were also explored based on the correlation analysis of the target microRNAs of these two circRNAs. In conclusion, we successfully established new PANC-1-GR cells, systemically characterized the circRNA and miRNA profiles, and identified two circRNAs as novel biomarkers and potential therapeutic targets for Gemcitabine non-responsive PDAC patients.

Keywords: pancreatic ductal adenocarcinoma, gemcitabine resistance, circular RNA, serum marker, diagnosis

\section{INTRODUCTION}

Pancreatic cancer is one of the most malignant cancers with very poor prognosis. The incidence of pancreatic cancer has been increasing over the past 20 years. $85 \%$ of pancreatic cancer is pancreatic ductal adenocarcinoma (PDAC) (Siegel et al., 2017). Although great progress has been made in surgery and other treatments for PDAC, the 5-year survival rate of PDAC is still less than $4 \%$, while 
the median survival of PDAC patients is only 5-6 months. Since most PDAC patients are diagnosed at an advanced stage, $80 \%$ of patients with PDAC is unable to undergo surgical resection treatment. Chemotherapy has become an essential treatment for advanced PDAC (Saung and Zheng, 2017). Gemcitabine is currently a first-line drug of chemotherapy treatment for PDAC patients. However, it is well known that only very few PDAC patients are able to maintain a lasting sensitivity to Gemcitabine-based chemotherapy (Amrutkar and Gladhaug, 2017). The drug resistance has already become the main reason for poor performance of Gemcitabine in the current treatment. Therefore, screening of Gemcitabine resistance related biological markers and improvement of Gemcitabine sensitivity are the main challenge of PDAC research.

Accumulating evidence has shown that circular RNAs (circRNAs), one of endogenous non-coding RNAs, play a key regulatory role in the cellular physiological process and the cancer biological process (Memczak et al., 2013). It is reported that circRNAs act as miRNA sponge which absorb miRNAs and then regulate the expression of miRNA targeted genes (Hansen et al., 2013a,b; Han et al., 2017). Most recently, circRNAs have been shown to be closely associated with various human diseases, such as cancer, cardiovascular disease and neurodegenerative diseases (Westholm et al., 2014; Wang et al., 2016; Chen et al., 2017a; Kristensen et al., 2017). In addition, the inherent stability of circRNAs conferred by the circular structure, allows them to be enriched in the exosomes and stably present in plasma, saliva and other peripheral tissues, which renders them potential diagnostic molecular markers for various diseases (Chen et al., 2017b; Wang et al., 2017).

In this study, we established a new Gemcitabine-resistant cell line (PANC-1-GR) from a pancreatic cancer cell line, PANC1. The circRNAs expression profile of PANC-1-GR cells was systematically explored comparing with parental PANC-1 cells by RNA sequencing. Our results showed that circRNAs expression profiles are very different between PANC-1 cells and PANC-1GR cells. The characterization of circRNAs and miRNAs in these cell lines and patient samples led to the identification of two novel circRNAs biomarkers and potential drug targets.

\section{MATERIALS AND METHODS}

\section{Patient Samples}

This study was approved by the Institutional Ethics Review Board of Anhui Provincial Hospital and was conducted according to the Ethical Guidelines for Human Genome/Gene Research issued by the Chinese Government. The plasma samples of PDAC patients were prospectively collected from 40 patients of Anhui Provincial Hospital from January 2015 to June 2016. All of the patients were histologically or cytologically confirmed as pancreatic ductal adenocarcinoma and received Gemcitabine-based chemotherapy. Twenty of these patients were found to be Gemcitabine non-responsive, as they meet the following definition: progression during or $<6$ months of previous Gemcitabine treatment including adjuvant therapy.

The peripheral blood $(5 \mathrm{ml})$ was collected in ethylenediaminetetraacetic acid tube, centrifuged at $3000 \mathrm{~g}$ for $10 \mathrm{~min}$ to harvest plasma, and stored at liquid nitrogen. Patients consent forms were duly signed by the patients according to the regulation of ethical guidelines issued by Anhui provincial hospital.

\section{Cell Lines}

PANC-1 cells were obtained from Shanghai Cell Bank. Cells were cultured in RPMI1640 supplemented in 10\% fetal bovine serum (FBS) with $100 \mathrm{U} / \mathrm{ml}$ penicillin-100 $\mathrm{g} / \mathrm{ml}$ streptomycin within a humidified incubator containing $5 \% \mathrm{CO}_{2}$ at $37^{\circ} \mathrm{C}$.

The cell line which was resistant to Gemcitabine was generated by selection under increasing gradient of Gemcitabine in our lab. The initial concentration of Gemcitabine in the cell culture medium was $0.1 \mu \mathrm{g} / \mathrm{ml}$. The concentration increased when the survival cells entered the logarithmic growth phase. After 40 weeks of continuous Gemcitabine induced culture, the final concentration of Gemcitabine in the resistant cell culture medium reach up to $200 \mu \mathrm{g} / \mathrm{ml}$. The Gemcitabine resistant cell line was named PANC-1-GR. The PANC-1-GR cell line was cultured in periodically added $10 \mu \mathrm{g} / \mathrm{ml}$ Gemcitabine to maintain cell resistance.

\section{Cell Viability and Proliferation Assays}

MTT assay was performed to determine cell viability of PANC1 and PANC-1-GR cell lines under Gemcitabine treatment and then indirectly reflected cell sensitivity to Gemcitabine. Cells seeded in 96-well plates, at a density of $5 \times 10^{4}$ cells per well, were given Gemcitabine treatment at different concentrations for $72 \mathrm{~h} .100 \mu \mathrm{l}$ of MTT solution $(500 \mu \mathrm{g} / \mathrm{ml}$ ) was added to each well and after its conversion to a soluble formazan, cell viability was measured by spectrophotometric absorbance at $570 \mathrm{~nm}$.

Cell proliferation was monitored with xCelligence system. PANC-1 and PANC-1-GR cells were seeded in a 96-well electronic microtiter plate (E-Plate), incubated at $37^{\circ} \mathrm{C}$ with $5 \% \mathrm{CO}_{2}$, and then monitored on the RTCA System at 30-min time interval for up to 100 hours. The electronic readout of cell-sensor impedance is displayed in realtime as cell index (CI), which directly influenced by cell attachment, spreading, or cell proliferation. The cell index is presented as mean $\pm \mathrm{SD}$ from three independent wells (calculated by xCELLigence) (Stefanowicz-Hajduk et al., 2016).

\section{RNA Extraction and Quality Control}

Total RNA was isolated from tumor tissues and plasma samples using TRIzol reagent (Invitrogen, Carlsbad, CA, United States), according to the manufacturer's protocol. RNA integrity was assessed using standard denaturing agarose gel electrophoresis. The total RNA from each specimen was quantified and quality assurance was provided by NanoDrop ND-1000 spectrophotometer (NanaDrop, Wilmington, DE, United States). 
TABLE 1 | Top 10 most differentially expressed circRNAs between PANC-1 and PANC-1-GR.

\begin{tabular}{|c|c|c|c|c|c|c|c|}
\hline circRNA & chrom & txStart & txEnd & Strand & CircBase ID & Gene Name & Regulation \\
\hline 1 & chr14 & 21825356 & 21829372 & - & hsa_circ_0000522 & SUPT16H & Up \\
\hline 2 & chr4 & 77045802 & 77065626 & - & hsa_circ_0070033 & NUP54 & Down \\
\hline 3 & chr1 & 15537793 & 155385714 & - & hsa_circ_0008161 & ASH1L & Down \\
\hline 4 & $\operatorname{chrX}$ & 154736558 & 154766779 & - & hsa_circ_0006355 & TMLHE & Down \\
\hline 5 & chr4 & 174305801 & 174325101 & + & Novel & SCRG1 & Up \\
\hline 6 & chr19 & 47421744 & 47440665 & + & hsa_circ_0000943 & ARHGAP35 & Up \\
\hline 7 & chr1 & 169947225 & 170001116 & - & Novel & KIFAP3 & Up \\
\hline 8 & chr14 & 101402108 & 101464448 & + & Novel & SNORD114-1 & Up \\
\hline 9 & chr4 & 52729602 & 52780244 & + & Novel & DCUN1D4 & Up \\
\hline 10 & chr6 & 29901994 & 29911250 & + & Novel & HLA-G & Up \\
\hline
\end{tabular}

\section{Transcriptome High-Throughput Sequencing and Subsequent Bioinformatics Analysis}

Transcriptome high-throughput sequencing and subsequent bioinformatics analysis were performed by Cloud-Seq Biotech (Shanghai, China). The RNA sequencing data had been deposited in the National Center for Biotechnology Information (NCBI) Gene Expression Omnibus (GEO). The GEO accession number is GSE110580 ${ }^{1}$. The scatter plot and cluster heatmap are visualization methods used for assessing the circRNA expression variation. The differentially expressed circRNA between PANC1 and Gemcitabine resistant PANC-1-GR cells were analyzed by edgeR package in R. Differentially expressed circRNAs with statistical significance (fold changes $\geq 1.5$ and $p<0.05$ ) between groups were identified using fold change cut-off or volcano plot filtering, respectively. The Database for Annotation, Visualization and Integrated Discovery (DAVID) bioinformatics tool for KEEG pathway enrichment analysis and Gene Ontology ${ }^{2}$, were applied to determine the roles that these differentially expressed circRNAs played in GO terms of biological pathways (Huang da et al., 2009). The circRNA/microRNA interaction was predicted using Arraystar's home-made miRNA target prediction software based on TargetScan and miRanda. The circRNAmiRNA network was constructed and visualized using Cytoscape v3.5.1 (Shannon et al., 2003).

\section{Quantitative Reverse Transcription-Polymerase Chain Reaction Validation Assay}

Total RNA samples were reverse-transcribed into cDNA with a random primer using SuperScript ${ }^{\mathrm{TM}}$ III Reverse Transcriptase (Invitrogen) according to the manufacturer's instructions. The expression of circRNAs was measured using quantitative polymerase chain reaction (qPCR) SYBR Green Master Mix (Takara, Tokyo, Japan) in a ViiA 7 Real-time PCR System (Applied Biosystems Inc., Foster City, CA, United States). The sequences of the divergent primers for the detection of the 10 circular RNAs by quantitative reverse transcription-polymerase chain reaction (qRT-PCR) were shown in Table 2. The RNA levels

${ }^{1}$ www.ncbi.nlm.nih.gov/geo/query/acc.cgi?acc=GSE110580

${ }^{2}$ https://david.ncifcrf.gov/ were normalized to human GAPDH. The expression levels were analyzed by the $2^{-\Delta \Delta \mathrm{Ct}}$ method.

All of the quantitative PCR reactions were conducted in triplicate. The appearance of a single-peak in the melt-curve suggested the specificity of the PCR products.

\section{Silencing and Over-Expression of circRNA}

siRNA sequences for chr14:101402109-101464448+ including: siRNA-1: CUUAAUUGUGGGCUCACAU; siRNA-2: CCUAUA GCUGUGGUAUAAC. siRNA sequences for chr4:52729603 -52780244+ including: siRNA-1: CAUAGUAAUAGACGAAU UGA; siRNA-2: UGAAUUCUUAGAAGUUAAAG.

siRNAs were synthesized by GeneChem (Shanghai, China).

The pCD-ciR plasmids was used to carry the circular framework of chr14:101402109-101464448+ and chr4:5272960352780244+. The primers for chr14:101402109-101464448+ are F: $5^{\prime}$-ATAAGTCTACTTTTCTTCCACGTAA- ${ }^{\prime}$

R: $\quad 5^{\prime}$-TTATASTGACATTCTCCTTACTCTGA-3'. The primers for chr4:52729603-52780244+ are F: 5'-CAGCT GAACTCTCATCTCTCAACAC-3'

R:5'-CCTTCCAGAAGTTGGCCTCTTAAAC-3'.

\section{Annexin V-FITC Cell Apoptosis Assay}

Cells were harvested for Annexin V-Propidium Iodide (PI) staining after $24 \mathrm{~h}$ with $0.1 \mathrm{ug} / \mathrm{ml}$ gemcitabine treatment. Cells were analyzed by the FACS Calibur (BD Biosciences). Annexin V-FITC ${ }^{+}$PI-cells were considered as apoptosis cells.

\section{TUNEL Assay}

Cells were exposed to $0.1 \mathrm{ug} / \mathrm{ml}$ gemcitabine for $24 \mathrm{~h}$, washed with PBS, and fixed in cold 4\% PFA for $30 \mathrm{~min}$, followed by incubation in $0.1 \%$ Triton X-100 in PBS for $2 \mathrm{~min}$ on ice. After washing twice in PBS, cells were incubated in working solutions from a One-Step TUNEL apoptosis assay kit (Beyotime Biotechnology).

\section{Statistical Analysis}

All experimental data were analyzed using SPSS software (version 22.0; IBM, Armonk, NY, United States) and GraghPad Prism 5.0 (GraphPad Software, La Jolla, CA, United States). The expression 
TABLE 2 | Primers used for qRT-PCR analysis of circular RNA and mRNA levels.

\begin{tabular}{|c|c|c|c|}
\hline CircRNA Name & Primer sequences, $5^{\prime}-3^{\prime}$ & $\operatorname{Tm}\left({ }^{\circ} \mathrm{C}\right)$ & PS (bp) \\
\hline \multirow[t]{2}{*}{ hsa_circ_0000522 } & F: ACTाGAGCGGGTCCAGTाT & 61.05 & 195 \\
\hline & R: TCTGAAGGTTCAAGGCTGGT & 59.84 & \\
\hline \multirow[t]{2}{*}{ hsa_circ_0070033 } & F: GCCAAAATTGCACAATACAAGA & 60.01 & 198 \\
\hline & R: TTGTGCCAAAACCAGTACCA & 60 & \\
\hline \multirow[t]{2}{*}{ hsa_circ_0008161 } & F: TTGGCTTAGTTGGATCCTCTG & 59.32 & 198 \\
\hline & R: TाTCCCTTGGGATGAGAGA & 59.6 & \\
\hline \multirow[t]{2}{*}{ hsa_circ_0006355 } & F: AGGCACCTGAGGAATाTGAA & 59.67 & 195 \\
\hline & R: TCCTITCTCCTGCCACATTC & 60.2 & \\
\hline \multirow[t]{2}{*}{ chr4:174305802-174325101+ } & F: GCTGTITCACAGACACAAGCA & 60.09 & 161 \\
\hline & R: CCCACGTTACTGAGCACAAA & 59.76 & \\
\hline \multirow[t]{2}{*}{ hsa_circ_0000943 } & F: GACAGAAACCAAAGCCCAAA & 60.09 & 197 \\
\hline & R: TGGTCACTGTTCACCACCTC & 59.55 & \\
\hline \multirow[t]{2}{*}{ chr1:169947226-170001116- } & F: ACCAGATGGTIITCCACCAA & 60.21 & 176 \\
\hline & R: CTITGTGCTITCCTCATTGC & 59.87 & \\
\hline \multirow[t]{2}{*}{ chr14:101402109-101464448+ } & F: CAGGATGGGTAGACCAGAGC & 59.68 & 182 \\
\hline & R: TACCCCACGGATCTAACTGC & 59.96 & \\
\hline \multirow[t]{2}{*}{ chr4:52729603-52780244+ } & F: TGGCATाTCTAGTCCCTाIT & 57.88 & 184 \\
\hline & R: TGCCAGTGTTGAGAGATGAGA & 59.56 & \\
\hline \multirow[t]{2}{*}{ chr6:29901995-29911250+ } & F: AAGGATTACATCGCCCTGAA & 59.53 & 198 \\
\hline & R: GTCCCTGGTACAGGTGTGCT & 60.03 & \\
\hline \multirow[t]{2}{*}{ GAPDH } & F: GGCCTCCAAGGAGTAAGACC & 60.07 & 122 \\
\hline & R: AGGGGAGATTCAGTGTGGTG & 59.96 & \\
\hline
\end{tabular}

Tm, melting temperature; PS, product size; bp, base pairs; F, Forward; R, Reverse

level of each circRNA was represented as fold-change using the $2^{-\Delta \Delta \mathrm{Ct}}$ method.

\section{RESULTS}

\section{Comparison of PANC-1 and PANC-1-GR Cell Lines}

A new PANC-1-GR cell line was derived from PANC-1 cell line by selecting under Gemcitabine gradients as shown in Section "Materials and Methods." The drug resistance of this new cell line was confirmed by culturing with Gemcitabine. After incubation with different concentrations of Gemcitabine $(0,0.1,0.5,2,10,20,40,80,120,160,200 \mu \mathrm{g} / \mathrm{ml})$ for $72 \mathrm{~h}$, cell viability was assessed by MTT assay. As shown in cell survival curves in Figure 1A, the 50\% inhibition concentrations $\left(\mathrm{IC}_{50}\right)$ of Gemcitabine to PANC-1 and PANC-G cells were $0.06 \pm 0.003 \mu \mathrm{g} / \mathrm{ml}$ and $56.2 \pm 2.16 \mu \mathrm{g} / \mathrm{ml}$, respectively. Cell proliferating ability of PANC-1 and PANC-1-GR cell lines was further monitored with xCelligence system. Cell growth data showed that PANC-1-GR cells proliferated slower than PANC1 cells (Figure 1B), which may be due to some cell cycle regulatory molecules differentially expressed between parental and resistant lines. And we will investigate the relevant cell cycle regulatory mechanisms in further study. We also determined the expression of the multidrug efflux pump MDR1, which is commonly observed to be upregulated in various drugs resistance cancer cells and also has been shown to cause gemcitabine resistance in pancreatic cancer cells. It was demonstrated that MDR1 expression was up-regulated in PANC-1-GR cells. (Supplementary Figure S1) The results confirmed that a PANC-1GR cell line was successfully established for subsequent circRNAs profiling.

\section{Characterization of circRNAs Profiles in PANC-1 and PANC-1-GR Cell Lines}

To screen circRNAs which could be involved in Gemcitabine resistance in PDAC, we analyzed and compared circRNAs expression in PANC-1 cells and PANC-1-GR cells using transcriptome high-throughput sequencing analysis. Total RNAs were isolated from PANC-1 and PANC-1-GR cell lines and analyzed by RNA sequencing. Differential gene expression analysis between PANC-1 and PANC-1-GR cells revealed 126 circRNAs whose expression was significantly different in these two cell lines (fold change $\geq 2.0, p \leq 0.05$ ), with 68 of them upregulated and 58 down-regulated in PANC-1-GR cells compared to PANC-1 cells (Figure 2).

\section{CircRNAs Gene Symbols and Pathway Analysis}

Recent studies have shown that circRNAs are derived from the exons or introns of their parental genes and may regulate the expression of the parental genes (Lasda and Parker, 2014). Based on evaluation of the parental genes attribute in the biological process, cellular components and molecular functions and pathways, we conducted GO and pathway analysis for circRNAs to speculate their potential functions. The lower the $p$ value was, the more significant 

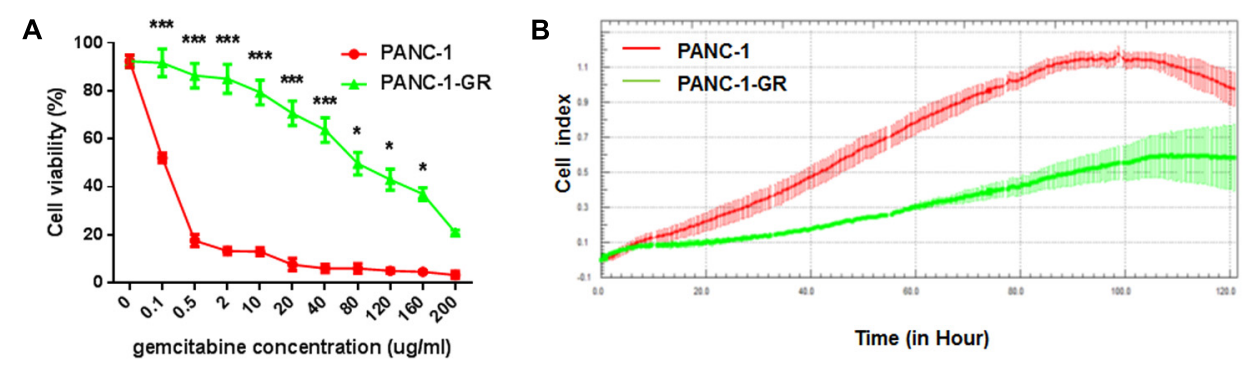

FIGURE 1 | Comparison of PANC-1 and PANC-1-GR cells. (A) Gemcitabine cytotoxicity to PANC-1 and PANC-1-GR cells. Cells were incubated continuously with different concentrations of Gemcitabine for $72 \mathrm{~h}$ and the cell viability was determined by MTT assay. (B) Proliferation assay of PANC-1 and PANC-1-GR cell lines. Data were collected from three independent cultures. Shown are mean values \pm standard deviation. ${ }^{* * *} p<0.001,{ }^{*} p<0.05$.
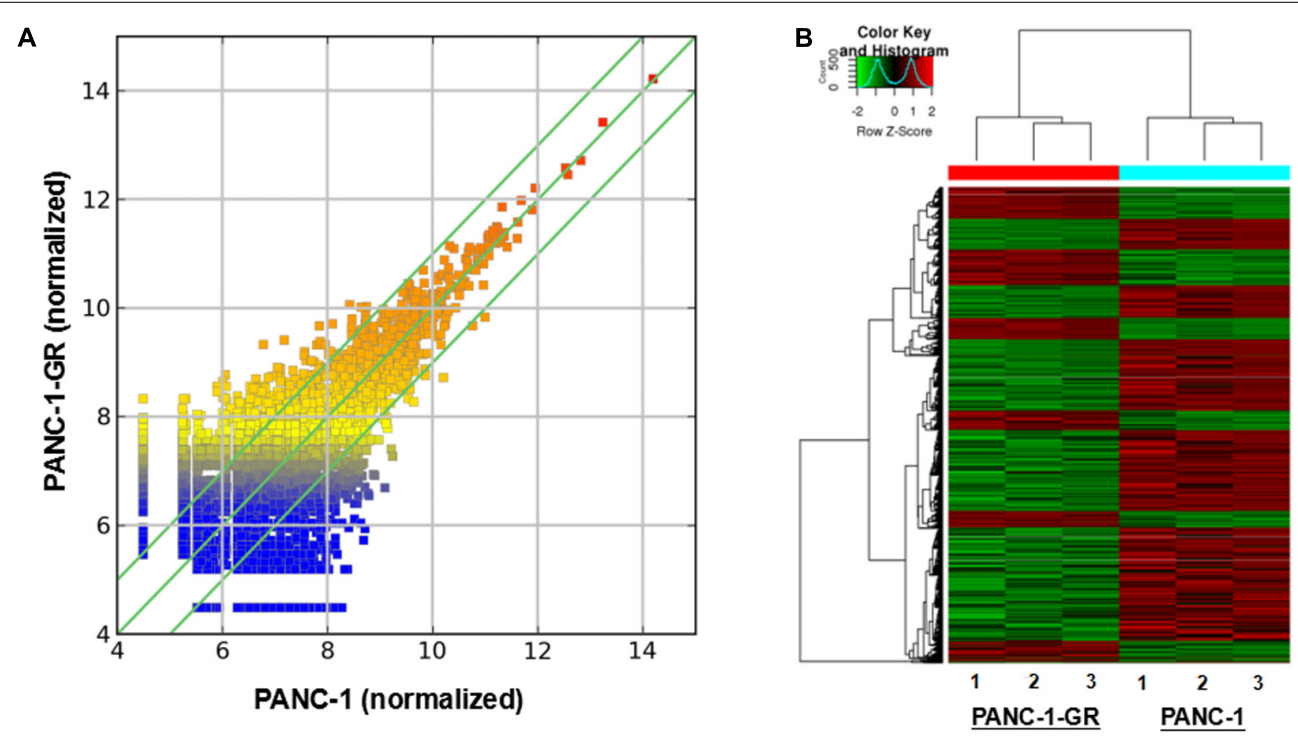

FIGURE 2 | circRNA expression profile of PANC-1-GR cells versus parental PANC-1 cells. (A) The scatter plot shows the circRNA expression variation between the parental PANC-1 and PANC-1-GR cell lines. The values of $X$ and $Y$ axes in the scatter plot are the averaged normalized signal values of groups of samples (log2 scaled). The green lines are fold change lines. The circRNAs above the top green line and below the bottom green line indicated more than 1.5-fold change of circRNAs between the two groups of samples. (B) Clustered heatmap of the differentially expressed circRNAs in three paired PANC-1 and PANC-1-GR cell lines. Rows represent circRNAs while columns represent cell lines. The circRNAs were classified according to the Pearson correlation.

the correlation was ( $p<0.05$ is recommended). We found that the most significantly enriched GO term in the biological process was the positive regulation of tolerance induction (GO:0002645, $P=0.0005$ ) (Figure 3A); the most significantly enriched GO term in the cellular component was protein complex (GO:0043234, $P=0.0001$ ) (Figure 3B); the most significantly enriched GO term in the molecular function was K63-linked polyubiquitin binding (GO:0070530, $P=0.0016$ ) (Figure 3C). Among the significantly related eight pathways, ErbB signaling pathway and VEGF signaling pathway were previously reported to be involved in the progression of PDAC (Figure 3D).

\section{Quantitative PCR Validation in Cell Lines}

To verify the sequencing results, the top 10 most differentially expressed circRNAs in PANC-1 and PANC1-GR cells including seven up-regulated circRNAs (hsa_circ_0000522, hsa_circ_0070033, hsa_circ_0000943, chr1:169947226-170001116-, chr14:101402109-101464448+, chr4:52729603-52780244+, and chr6:29901995-29911250+) and three down-regulated circRNAs (hsa_circ_0070033, hsa_circ_0008161, and hsa_circ_0006355) (Table 1) were further confirmed by qRT-RCR. The qRT-PCR data showed that although the trend of expression patterns of these 10 circRNAs were consistent with the sequencing results, among these circRNAs, only two of them (chr14:101402109101464448+, chr4:52729603-52780244+) were found to be the most significantly up-regulated in PANC-1-GR cell line (Figure 4).

\section{Expression of circRNAs in Plasma of PDAC Patients}

Subsequently, we verified the expression levels of these two most significant circRNAs (chr14:101402109-101464448+, 

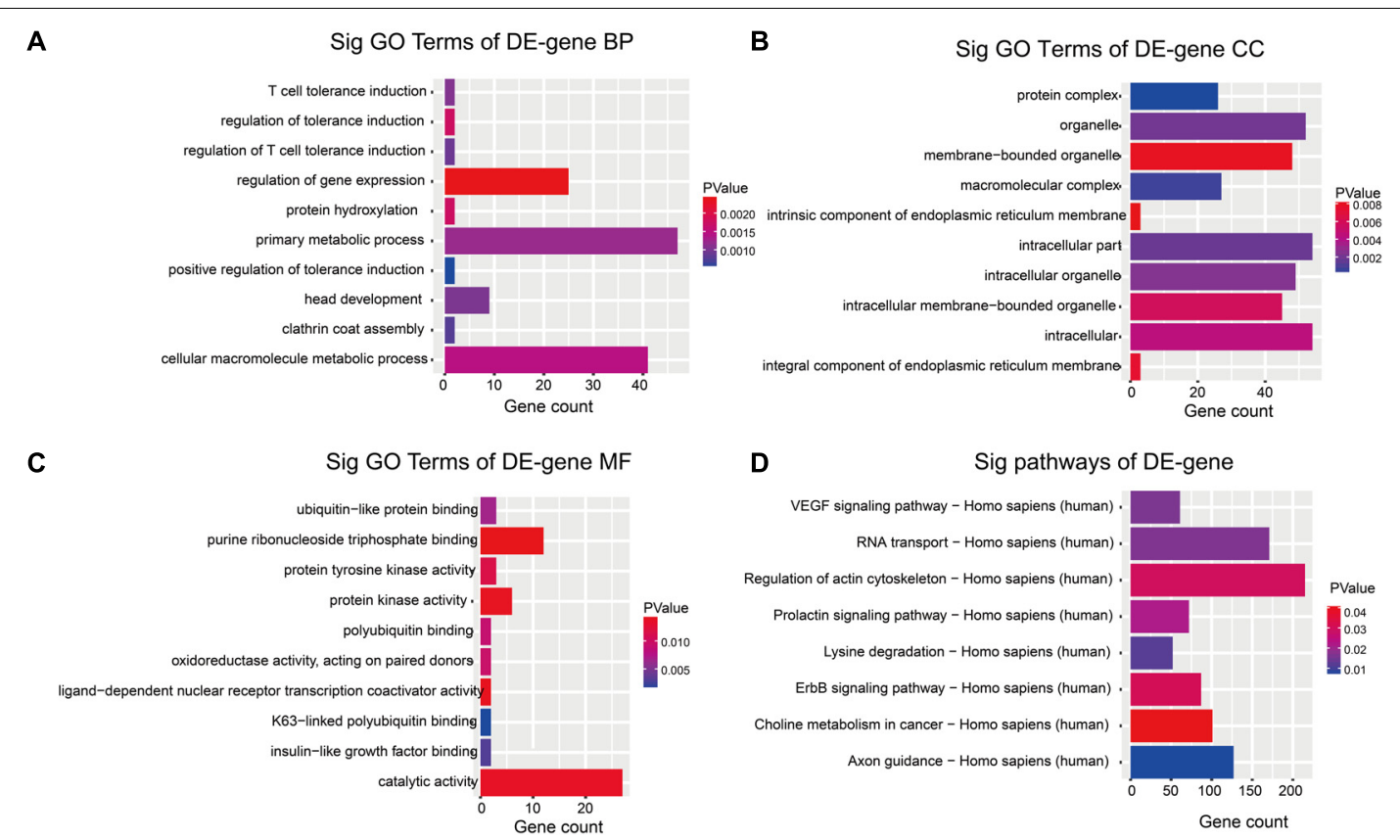

FIGURE 3 | GO enrichment and pathway analysis for dysregulated circRNAs gene symbols. (A) Most significantly enriched GO [-log10 (P value)] terms of circRNAs gene symbols according to biological process. (B) Most significantly enriched [-log10 ( $P$ value)] GO terms of circRNAs gene symbols according to cellular component. (C) Most significantly enriched [-log10 (P value)] GO terms of circRNAs gene symbols according to molecular function. (D) The bar plot shows the top 10 enrichment score $[-\log 10(P$ value $)]$ of the significantly enriched pathways.

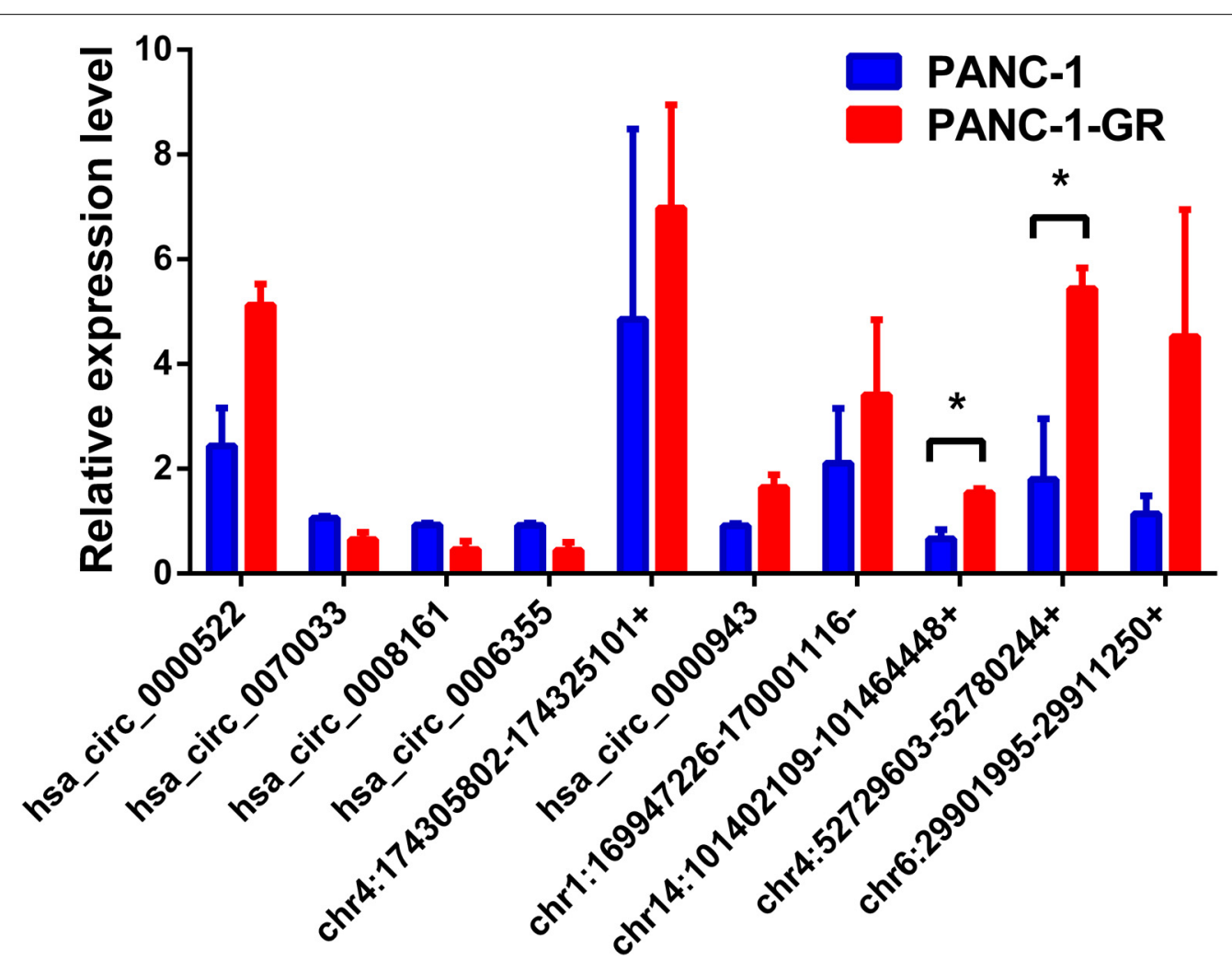

FIGURE 4 | Validation of the top 10 dysregulated circRNAs by quantitative RT-PCR analysis. The top 10 most differentially expressed circRNAs were validated by qRT-PCR in PANC-1 and PANC-1-GR cell lines. The results are presented as mean \pm SEM. ${ }^{*} p<0.05$. 
chr4:52729603-52780244+) in plasma of PDAC patients who received Gemcitabine-based chemotherapy via qRT-PCR. In addition, hsa_circ_0008161, which was not significantly regulated as in qRT-PCR data, was used as a negative control. The Gemcitabine-treated PDAC patients were divided into responsive and non-responsive groups according to the effect of Gemcitabine treatment. The clinical characteristics of the patients were shown in Table 3. Consistent to RNA sequencing and qRT-PCR data from PANC-1-GR cells, chr14:101402109101464448+ and chr4:52729603-52780244+ were found significantly up-regulated in non-responsive group $(p<0.001)$, while there was no significant difference in hsa_circ_0008161 (Figure 5).

\section{Network of circRNAs and the Predicted Binding miRNAs}

To better explore and understand the upstream and downstream miRNAs associated to the two circRNAs, we analyzed the potential binding miRNAs for the two circRNAs by sequence analysis with TargetScan. A tree diagram of circRNAs and their potential binding miRNAs is generated in Figure 6A. Based on circRNA/miRNA interaction network, chr14:101402109101464448+ and chr4:52729603-52780244+ were predicted to be able to bind a spectrum of miRNAs with known functions, suggesting their potential roles in Gemcitabine resistance of PDAC. We selected three potential target miRNAs from the tree diagram, including miR-19a-3p, miR-138-5p, and

TABLE 3 | Clinical characteristics of the patients.

\begin{tabular}{|c|c|c|c|c|}
\hline $\begin{array}{l}\text { Clinicopathologic } \\
\text { factors }\end{array}$ & $n$ & Responsive & Non-responsive & $P$-value \\
\hline Age & & & & 0.519 \\
\hline$<60$ years & 24 & 11 & 13 & \\
\hline$\geq 60$ years & 16 & 9 & 7 & \\
\hline Sex & & & & 0.256 \\
\hline Male & 31 & 14 & 17 & \\
\hline Female & 9 & 6 & 3 & \\
\hline Tumor location & & & & 0.376 \\
\hline Head & 34 & 18 & 16 & \\
\hline Body/tail & 6 & 2 & 4 & \\
\hline Serum CA19-9 & & & & 0.633 \\
\hline$\leq 37 \mathrm{U} / \mathrm{mL}$ & 5 & 3 & 2 & \\
\hline$>37 \mathrm{U} / \mathrm{mL}$ & 35 & 17 & 18 & \\
\hline $\begin{array}{l}\text { Number of } \\
\text { metastatic lesions }\end{array}$ & & & & 0.376 \\
\hline 1 & 6 & 2 & 4 & \\
\hline$\geq 2$ & 34 & 18 & 16 & \\
\hline $\begin{array}{l}\text { Karnofsky } \\
\text { performance status } \\
\text { score }\end{array}$ & & & & 0.248 \\
\hline 100 & 7 & 4 & 3 & \\
\hline 90 & 8 & 3 & 5 & \\
\hline 80 & 19 & 10 & 9 & \\
\hline 70 & 3 & 2 & 1 & \\
\hline $50-60$ & 3 & 1 & 2 & \\
\hline
\end{tabular}

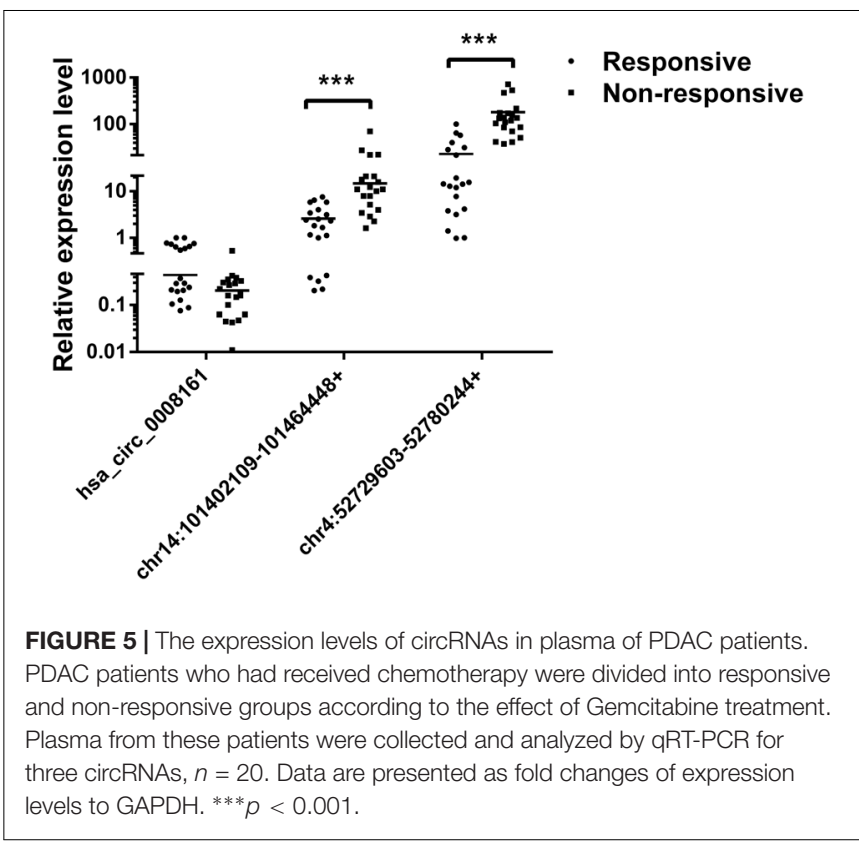

miR-145-5p, which may bind both of the two circRNAs, and compare the expression of them between PANC-1 and PANC-1-GR cells. It was found that miR-19a-3p and miR-145-5p were down-regulated in PANC-1-GR cells, while miR-138-5p expression did not change significantly (Figure 6B). We also analyzed the miR-145-5p expression of plasma of PDAC patients who received gemcitabine treatment. It was found miR-145-5p was down-regulated in non-responsive group, compared with responsive group (Figure 6C).

\section{Silencing of circRNAs Enhances Gemcitabine Sensitivity of PANC-1-GR}

To evaluate the functions of these two new circRNAs in Gemcitabine resistance, we applied small RNA interference (siRNAs) to silence the expression of chr14:101402109$101464448+$ and chr4:52729603-52780244+ in PANC-1-GR cells. Two siRNAs were designed to target the backsplice sequence of each circRNA, respectively. A non-specific control siRNA sequence was also used as the negative control. After transfection, siRNA1 of chr14:101402109-101464448+ and siRNA2 of chr4:52729603-52780244+, dramatically inhibited the expression of chr14:101402109-101464448+ and chr4:5272960352780244+, respectively, in PANC-1-GR cells (Figure 7A), which were further used for transfection in cytotoxicity assay. It was demonstrated that after silencing of chr14:101402109101464448+ or chr4:52729603-52780244+, PANC-1-GR cells restored sensitivity to Gemcitabine (Figure 7B). Annexin V staining apoptosis assay also demonstrated that siRNA group had more Annexin $\mathrm{V}$ positive apoptosis cells after cells were cultured with $0.1 \mathrm{ug} / \mathrm{ml}$ gemcitabine for $24 \mathrm{~h}$ (Figure 7C). These results suggested that the two circRNAs may serve as potential therapeutic targets for Gemcitabine resistance in PDAC. 

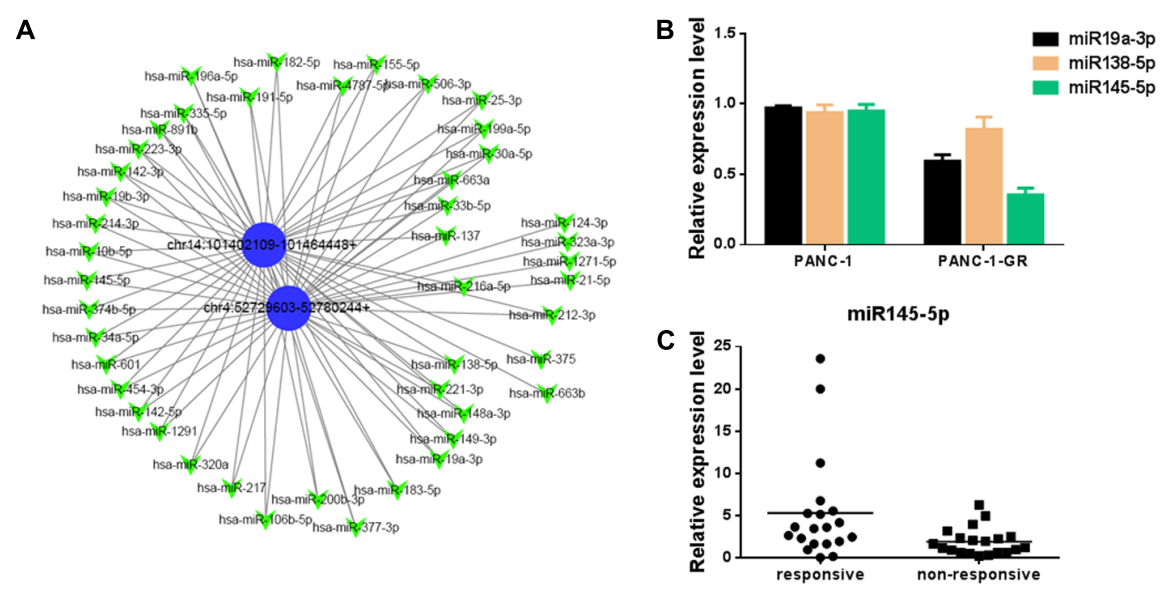

FIGURE 6 | Network of circular RNAs and the predicted binding miRNAs. (A)The two circRNAs biomarkers were annotated in detail according to the circRNA/miRNA interaction information by Cytoscape. (B) miR19a-3p, miR138-5p and miR145-5p expression in PANC-1 and PANC-1-GR cells. (C) miR145-5p expression in plasma of PDAC patients who received gemcitabine treatment, including responsive and non-responsive groups.

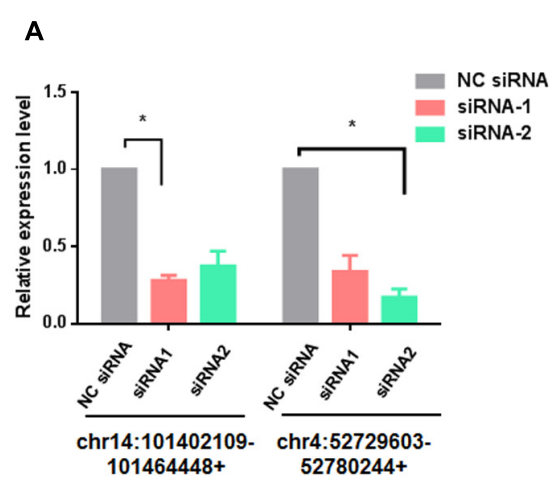

B
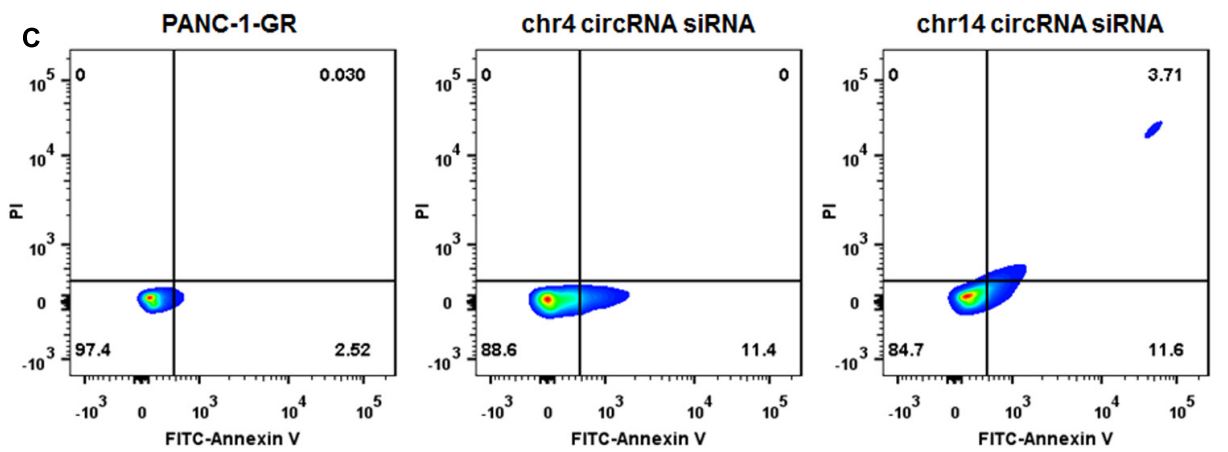

FIGURE 7 | Silencing of circRNAs enhances Gemcitabine sensitivity of PANC-1-GR cell lines. (A) qRT-PCR analysis of circRNA expression level after siRNAs transfection. NC siRNA: negative control siRNA. (B) Cell viability of PANC-1-GR cells after transfection with siRNAs. Cells were incubated with different concentrations of Gemcitabine for $72 \mathrm{~h}$ and then cell viability was determined by MTT assay. ${ }^{*} p<0.05$. (C) Annexin V-FITC staining assay was performed for PANC-1-GR cells and siRNA tranfected PANC-1-GR cells.

\section{Overexpression of circRNAs Enhances Gemcitabine Resistance of PANC-1 and MIA PACA-2 Cells}

We also overexpressed the circRNAs in parental PANC1 and MIA PACA-2 cells. pCD-ciR plasmids was used to carry the circular framework of chr14:101402109-101464448+ and chr4:52729603-52780244+. After transfection and overexpression of chr14:101402109-101464448+ and chr4:52729603$52780244+$ in PANC-1 cells, PANC-1 cells showed more resistant to gemcitabine, with less Annexin $\mathrm{V}$ positive cells after $0.1 \mathrm{ug} / \mathrm{ml}$ gemcitabine treatment for $24 \mathrm{~h}$ (Figure 8A and Supplementary Figure S2). The same result can be observed 

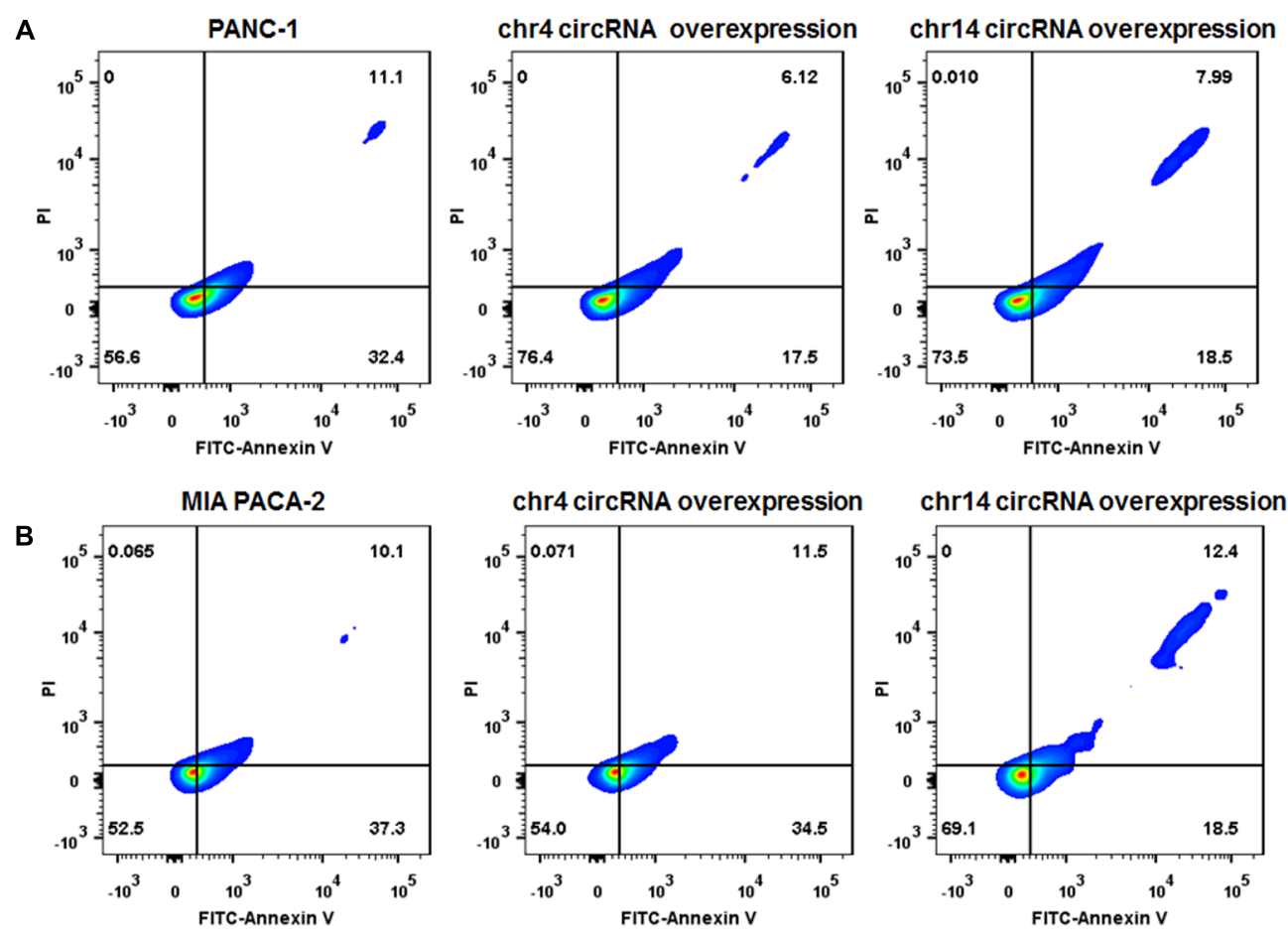

FIGURE 8 | Over-expression of circRNAs enhances Gemcitabine resistance of PANC-1 and MIA PACA-2 cells. (A) Annexin V-FITC staining assay was performed for PANC-1 cells, chr4 circRNA and chr14 circRNA tranfected PANC-1 cells. Cells were incubated with $0.1 \mathrm{ug} / \mathrm{ml}$ of Gemcitabine for $24 \mathrm{~h}$. (B) Annexin V-FITC staining assay was performed for MIA PACA-2 cells, chr4 circRNA tranfected and chr14 circRNA tranfected MIA PACA-2 cells. Cells were incubated with 0.1 ug/ml of Gemcitabine for $24 \mathrm{~h}$.

in MIA PACA-2 cells, when tranfected with chr14:101402109$101464448+$ circular framework (Figure 8B and Supplementary Figure S2). However, only overexpression of chr14:101402109$101464448+$ was able to exert some resistant phenotype to Gemcitabine in MIA PACa-2 while chr4:52729603-52780244+ was not. The downstream regulatory function of chr4:52729603$52780244+$ may be compensated or antagonized by other regulatory mechanisms in MIA PACA-2 cells. We also analyzed miR-145 expression in parental PANC-1 and MIA PACA2 cells when they overexpressed chr4:52729603-52780244+ and chr14:101402109-101464448+. It was found that miR-145 expression decreased to different extents, when chr4:5272960352780244+ and chr14:101402109-101464448+ overexpressed in parental PANC-1 and MIA PACA-2 cells. (Supplementary Figure S3) It further suggests that miR145 may be involved in gemcitabine resistance by circRNA-miRNA interaction.

\section{DISCUSSION}

In this study, we developed Gemcitabine resistant pancreatic cancer cell line PANC-1-GR as a research tool to investigate Gemcitabine resistance. Subsequently, we compared the differences of circRNAs expression profile between PANC-1 and PANC-1-GR cell lines using RNA sequencing analysis. From the sequencing data, it was found that there were 68 up-regulated circRNAs and 58 down-regulated circRNAs which are possibly related to Gemcitabine resistance in PANC-1-GR. Upon validating the top 10 dys-regulated circRNAs using qRT-PCR in these cell lines, it is interesting to see that RNA sequencing data was quite consistent with qRT-PCR and combining both, we were able to identify two most distinctly expressed circRNAs from PANC-1-GR cells when compared to PANC-1 cells. It is even more exciting to confirm that these two markers are also consistently found to be highly expressed in the plasma from Gemcitabine non-responsive PDAC patients but not in Gemcitabine responsive ones. Our study demonstrated that the two circRNAs may be functionally involved in generating Gemcitabine resistance as the silencing of them can restore the sensitivity of PANC-1-GR cells to Gemcitabine.

Besides, GO and pathway analysis of circRNAs patent genes were investigated. GO enrichment analysis revealed that some gene symbols were involved in the regulation of biological process, cellular component and molecular function. Among the GO terms found in this study, "primary metabolic process" and "insulin-like growth factor binding" may play important roles in the drug resistance of PANC-1 cells via drug metabolic or cell proliferation pathway (Ireland et al., 2016; Chen et al., 2017c). Meanwhile, "ErbB signaling pathway" has been reported to be involved in Gemcitabine resistance of pancreatic cancer (Skrypek et al., 2015) and "VEGF signaling pathway" has been reported to be involved in the progression of pancreatic cancer, which may contribute to cell drug resistance (Zhou et al., 2016; Zhu et al., 2016). These findings indicated that in 
addition to the two biomarkers identified in this study, there could be more circRNAs involved in the Gemcitabine resistance of PANC-1-GR cells. Recent studies have demonstrated that circRNAs could regulate gene expression as miRNA sponges or potent competitive endogenous RNA (ceRNA) molecules ( $\mathrm{Qu}$ et al., 2017). Given that miRNAs play important roles in the Gemcitabine resistance of pancreatic cancer (Amponsah et al., 2017; Chaudhary et al., 2017; Xiong et al., 2017), some circRNAs could likely be involved in Gemcitabine resistance via interacting with miRNAs. We found that the majority of circRNAs contained one or more miRNA binding sites based on the sequences analysis. Since we identify the two circRNAs markers in this study, preparing more information about miRNA networks of these two circRNAs is meaningful as it may lead to a better understanding of their upstream and downstream miRNA targets which could serve as potential markers and drug targets. The association of miRNAs with PDAC indicated that circRNAs may have a regulatory role in PDAC. For example, among the founded potential circRNA/miRNA interactions, chr4:52729603$52780244+$ is potentially able to bind miR124-3p, which has been reported playing a critical role in Gemcitabine resistance of pancreatic cancer ( $\mathrm{Li}$ et al., 2016). MiR-145, which may bind to both chr14:101402109-101464448+ and chr4:52729603$52780244+$, was also known to be associated with the resistance of pancreatic cancer cells to Gemcitabine (Zhuang et al., 2017). It was found that MiR-145-5p was down-regulated in both PANC1-GR cells and plasma of non-responsive patients. Certainly, future studies are required to clarify the underlying mechanism of these circRNAs-miRNA interactions in Gemcitabine resistance of pancreatic cancer.

In conclusion, our study provides a new research tool, PANC-1-GR cell line and based on this tool, new insights into Gemcitabine resistance in pancreatic cancer treatment by identifying two novel circRNAs biomarkers and drug targets.

\section{REFERENCES}

Amponsah, P. S., Fan, P., Bauer, N., Zhao, Z., Gladkich, J., Fellenberg, J., et al. (2017). microRNA-210 overexpression inhibits tumor growth and potentially reverses gemcitabine resistance in pancreatic cancer. Cancer Lett. 388, 107-117. doi: 10.1016/j.canlet.2016.11.035

Amrutkar, M., and Gladhaug, P. I. (2017). Pancreatic cancer chemoresistance to gemcitabine. Cancers 9:E157. doi: 10.3390/cancers9110157

Chaudhary, A. K., Mondal, G., Kumar, V., Kattel, K., and Mahato, R. I. (2017). Chemosensitization and inhibition of pancreatic cancer stem cell proliferation by overexpression of microRNA-205. Cancer Lett. 402, 1-8. doi: 10.1016/j. canlet.2017.05.007

Chen, J., Cui, L., Yuan, J., Zhang, Y., and Sang, H. (2017a). Circular RNA WDR77 target FGF-2 to regulate vascular smooth muscle cells proliferation and migration by sponging miR-124. Biochem. Biophys. Res. Commun. 494, 126-132. doi: 10.1016/j.bbrc.2017.10.068

Chen, J., Li, Y., Zheng, Q., Bao, C., He, J., Chen, B., et al. (2017b). Circular RNA profile identifies circPVT1 as a proliferative factor and prognostic marker in gastric cancer. Cancer Lett. 388, 208-219. doi: 10.1016/j.canlet.2016.12.006

Chen, R., Lai, L. A., Sullivan, Y., Wong, M., Wang, L., Riddell, J., et al. (2017c). Disrupting glutamine metabolic pathways to sensitize gemcitabine-resistant pancreatic cancer. Sci. Rep. 7:7950. doi: 10.1038/s41598-017-08436-6

Han, D., Li, J., Wang, H., Su, X., Hou, J., Gu, Y., et al. (2017). Circular RNA circMTO1 acts as the sponge of microRNA-9 to suppress hepatocellular carcinoma progression. Hepatology 66, 1151-1164. doi: 10.1002/hep.29270
Future investigation should be followed up focusing on the exploration of underlying mechanism of the two circRNAs and their associated networks. We hope this work would help to accelerate the development of novel therapeutic strategies targeting Gemcitabine-based chemotherapy of PDAC patients.

\section{AUTHOR CONTRIBUTIONS}

FS and $\mathrm{MH}$ carried out the experiments and drafted the initial manuscript. FM contributed to the literature search and bioinformatic analysis. $\mathrm{QH}$ reviewed the statistics and contributed to critical revisions. All authors reviewed and approved the final manuscript as submitted.

\section{FUNDING}

This study was supported by grants from the National Natural Science Foundation of China (81501354).

\section{ACKNOWLEDGMENTS}

We thank the other members of Anhui Province Key Laboratory of Hepatopancreatobiliary Surgery for providing constructive advice in study design and data analysis.

\section{SUPPLEMENTARY MATERIAL}

The Supplementary Material for this article can be found online at: https://www.frontiersin.org/articles/10.3389/fphar. 2018.00584/full\#supplementary-material

Hansen, T. B., Jensen, T. I., Clausen, B. H., Bramsen, J. B., Finsen, B., Damgaard, C. K., et al. (2013a). Natural RNA circles function as efficient microRNA sponges. Nature 495, 384-388. doi: 10.1038/nature 11993

Hansen, T. B., Kjems, J., and Damgaard, C. K. (2013b). Circular RNA and miR-7 in cancer. Cancer Res. 73, 5609-5612. doi: 10.1158/0008-5472.CAN-13-1568

Huang da, W., Sherman, B. T., and Lempicki, R. A. (2009). Systematic and integrative analysis of large gene lists using DAVID bioinformatics resources. Nat. Protoc. 4, 44-57. doi: 10.1038/nprot.2008.211

Ireland, L., Santos, A., Ahmed, M. S., Rainer, C., Nielsen, S. R., Quaranta, V., et al. (2016). Chemoresistance in pancreatic cancer is driven by stroma-derived insulin-like growth factors. Cancer Res. 76, 6851-6863. doi: 10.1158/0008-5472. CAN-16-1201

Kristensen, L. S., Hansen, T. B., Venø, M. T., and Kjems, J. (2017). Circular RNAs in cancer: opportunities and challenges in the field. Oncogene 37, 555-565. doi: $10.1038 /$ onc.2017.361

Lasda, E., and Parker, R. (2014). Circular RNAs: diversity of form and function. RNA 20, 1829-1842. doi: 10.1261/rna.047126.114

Li, C., Zhao, Z., Zhou, Z., and Liu, R. (2016). Linc-ROR confers gemcitabine resistance to pancreatic cancer cells via inducing autophagy and modulating the miR-124/PTBP1/PKM2 axis. Cancer Chemother. Pharmacol. 78, 1199-1207. doi: 10.1007/s00280-016-3178-4

Memczak, S., Jens, M., Elefsinioti, A., Torti, F., Krueger, J., Rybak, A., et al. (2013). Circular RNAs are a large class of animal RNAs with regulatory potency. Nature 495, 333-338. doi: 10.1038/nature 11928 
Qu, S., Zhong, Y., Shang, R., Zhang, X., Song, W., Kjems, J., et al. (2017). The emerging landscape of circular RNA in life processes. RNA Biol. 14, 992-999. doi: 10.1080/15476286.2016.1220473

Saung, M. T., and Zheng, L. (2017). Current standards of chemotherapy for pancreatic cancer. Clin. Ther. 39, 2125-2134. doi: 10.1016/j.clinthera.2017. 08.015

Shannon, P., Markiel, A., Ozier, O., Baliga, N. S., Wang, J. T., Ramage, D., et al. (2003). Cytoscape: a software environment for integrated models of biomolecular interaction networks. Genome Res. 13, 2498-2504. doi: 10.1101/ gr.1239303

Siegel, R. L., Miller, K. D., and Jemal, A. (2017). Cancer statistics, 2017. CA Cancer J. Clin. 67, 7-30. doi: 10.3322/caac.21387

Skrypek, N., Vasseur, R., Vincent, A., Duchêne, B., Van Seuningen, I., and Jonckheere, N. (2015). The oncogenic receptor ErbB2 modulates gemcitabine and irinotecan/SN-38 chemoresistance of human pancreatic cancer cells via hCNT1 transporter and multidrug-resistance associated protein MRP-2. Oncotarget 6, 10853-10867. doi: 10.18632/oncotarget. 3414

Stefanowicz-Hajduk, J., Adamska, A., Bartoszewski, R., and Ochocka, J. R. (2016). Reuse of E-plate cell sensor arrays in the xCELLigence Real-Time Cell Analyzer. Biotechniques 61, 117-122. doi: 10.2144/000114450

Wang, J., Yu, Y., Li, G., Shen, C., Meng, Z., Zheng, J., et al. (2017). Relationship between serum HBV-RNA levels and intrahepatic viral as well as histologic activity markers in entecavir-treated patients. J. Hepatol. doi: 10.1016/j.jhep. 2017.08.021 [Epub ahead of print].

Wang, K., Long, B., Liu, F., Wang, J. X., Liu, C. Y., Zhao, B., et al. (2016). A circular RNA protects the heart from pathological hypertrophy and heart failure by targeting miR-223. Eur. Heart J. 37, 2602-2611. doi: 10.1093/eurheartj/ehv713

Westholm, J. O., Miura, P., Olson, S., Shenker, S., Joseph, B., Sanfilippo, P., et al. (2014). Genome-wide analysis of drosophila circular RNAs reveals their structural and sequence properties and age-dependent neural accumulation. Cell Rep. 9, 1966-1980. doi: 10.1016/j.celrep.2014.10.062

Xiong, J., Wang, D., Wei, A., Ke, N., Wang, Y., Tang, J., et al. (2017). MicroRNA410-3p attenuates gemcitabine resistance in pancreatic ductal adenocarcinoma by inhibiting HMGB1-mediated autophagy. Oncotarget 8, 107500-107512. doi: 10.18632 /oncotarget.22494

Zhou, R., Curry, J. M., Roy, L. D., Grover, P., Haider, J., Moore, L. J., et al. (2016). A novel association of neuropilin-1 and MUC1 in pancreatic ductal adenocarcinoma: role in induction of VEGF signaling and angiogenesis. Oncogene 35, 5608-5618. doi: 10.1038/onc.2015.516

Zhu, M., Zhang, Q., Wang, X., Kang, L., Yang, Y., Liu, Y., et al. (2016). Metformin potentiates anti-tumor effect of resveratrol on pancreatic cancer by down-regulation of VEGF-B signaling pathway. Oncotarget 7, 84190-84200. doi: 10.18632/oncotarget.12391

Zhuang, J., Shen, L., Yang, L., Huang, X., Lu, Q., Cui, Y., et al. (2017). TGFbetal promotes gemcitabine resistance through regulating the LncRNALET/NF90/miR-145 signaling axis in bladder cancer. Theranostics 7, 3053-3067. doi: 10.7150/thno.19542

Conflict of Interest Statement: The authors declare that the research was conducted in the absence of any commercial or financial relationships that could be construed as a potential conflict of interest.

Copyright (c) 2018 Shao, Huang, Meng and Huang. This is an open-access article distributed under the terms of the Creative Commons Attribution License (CC BY). The use, distribution or reproduction in other forums is permitted, provided the original author(s) and the copyright owner are credited and that the original publication in this journal is cited, in accordance with accepted academic practice. No use, distribution or reproduction is permitted which does not comply with these terms. 